\title{
Investigation of Anti-SARS-CoV-2 IgG and IgM Antibodies in the Patients with COVID-19 by Three Different ELISA Test Kits
}

\author{
Masayuki Nagasawa ${ }^{1,2}$ (1) $\cdot$ Yoshimi Yamaguchi $^{3} \cdot$ Maki Furuya $^{3} \cdot$ Yui Takahashi $^{3} \cdot$ Reiko Taki $^{4} \cdot$ Kaoru Nagata $^{5} \cdot$ \\ Shinichiro Suzaki ${ }^{6} \cdot$ Masayuki Kurosaki $^{7} \cdot$ Namiki Izumi $^{8}$
}

Accepted: 10 July 2020 / Published online: 23 July 2020

(C) Springer Nature Switzerland AG 2020

\begin{abstract}
We examined anti-SARS-CoV-2 IgG and IgM antibodies in 45 serum samples from 26 patients with COVID-19, who were admitted in our hospital by using three different ELISA kits. All patients had pneumonia at admission, and 7 patients required mechanical ventilator support and grouped in severe case. Anti-SARS-CoV-2 IgG and IgM antibodies turned to be partially positive between the 6 th and 10th days, more than $84 \%$ positive between the 11 th and 15 th days, and $100 \%$ after the 16th day. One ELISA kit revealed poorer sensitivity for anti-SARS-CoV-2 IgM antibody. Negative conversion of IgM antibody was not observed in the 30th day in our cohort. All three ELISA kits showed no false positive reaction for negative serum samples. Between severe and moderate cases, there was no significant difference in the trends of anti-SARS-CoV-2 IgG and IgM antibody.
\end{abstract}

Keywords SARS-CoV-2 COVID-19 · ELISA · Antibody

\section{Introduction}

In December 2019, novel coronavirus (SARS-CoV-2) infection (COVID-19) has emerged in China and spread with amazing speed worldwide and resulted in the pandemic [1]. The pandemic has deep and serious impact not only on the medical service but also on socio-economic activity worldwide. More than 7 million people were infected, and more than 400,000 people were dead in the world at the moment of early June 2020 [2]. In addition to the absence of specific treatment and effective vaccine, it makes infection control very difficult that there are many infected patients with no symptom and slight illness, who spread virus into the society insidiously.

The Japanese Government pronounced emergency declaration on April 7, and infection eventually burnt down in 2 months. However, the problem in the future infection control for the 2nd wave of COVID-19 is not solved fundamentally yet. In this context, the epidemiological analysis in the society by using the test for antibodies is expected to give important information for the future public health strategy.

According to the concept above, we have investigated antiSARS-CoV-2 antibody in hospitalized infected patients in our

This article is part of the Topical Collection on Covid-19

Masayuki Nagasawa

mnagasawa.ped@tmd.ac.jp

1 Department of Infection Control, Musashino Red Cross Hospital, 1-26-1, Kyonan-cho, Musashino City, Tokyo 180-8610, Japan

2 Department of Pediatrics, Musashino Red Cross Hospital, 1-26-1, Kyonan-cho, Musashino City, Tokyo 180-8610, Japan

3 Department of Laboratory, Musashino Red Cross Hospital, Musashino City, Japan
4 Department of Respiratory Medicine, Musashino Red Cross Hospital, Musashino City, Japan

5 Department of General Medicine, Musashino Red Cross Hospital, Musashino City, Japan

6 Department of Emergency and Intensive Care, Musashino Red Cross Hospital, Musashino City, Japan

7 Department of Gastroenterology and Hepatology, Musashino Red Cross Hospital, Musashino City, Japan

8 Musashino Red Cross Hospital, 1-26-1, Kyonancho, Musashino City, Tokyo 180-8610, Japan 
Table 1 The summary of the patients in this cohort

\begin{tabular}{ll}
\hline Patients & 26 (male 14, female 12) \\
Age (years) & $19 \sim 82(52.6 \pm 16.3)$ \\
Pneumonia & 26 \\
Ventilator usage & 7 \\
WBC $(/ \mu \mathrm{L})$ & $2700 \sim 11900(5946 \pm 2279)$ \\
CRP $(\mathrm{mg} / \mathrm{dL})$ & $0.02 \sim 28.34(6.63 \pm 7.63)$ \\
Death & 1 \\
\hline
\end{tabular}

institute by using three different enzyme-linked immunosorbent assay (ELISA) kits.

\section{Patients and Methods}

Twenty six patients who were diagnosed as COVID-19 from positive RT-PCR test for SARS-CoV-2 by using naso-pharynx swab specimens and admitted in our hospital between April 12 and May 8, 2020 were involved in this study. RT-PCR test was performed at SRL laboratory (Tokyo, Japan). All patients had fever at admission and received chest computed tomography (CT) examination to detect pneumonia. The initial day with a temperature of $37.5^{\circ} \mathrm{C}$ and more was determined as a first day of the disease. A total of 45 serum samples from 26 COVID-19 patients obtained between the 1st and 29th days of the disease were used for this study. As negative controls, we used serum samples from 12 patients who were admitted during the same period because of pneumonia and confirmed not to be as COVID19 because of negative RT-PCR test for SARS-CoV-2 and the comprehensive clinical evaluations. This study was approved by the institutional review board as No2010. The written informed consent was waived for emerging infectious disease, and informed consent was secured by opt-out manner.

Statistical analysis was performed by Mann-Whitney $U$ test, and $p<0.05$ was considered as significant.

\section{ELISA Test for Anti-SARS-CoV-2 IgG and IgM Antibodies}

ELISA tests used in this study were 2019-nCoV Ab Test (INNOVITA Biological Technology Co., China), COVID19 IgG/IgM Rapid Test Cassette (Zhejiang Orient Gene Biotech Co., China), and 2019-nCoV IgG/IgM Rapid Test Cassette (Hangzhou AllTest Biotech Co., China), which were kindly provided by SoftBank Cooperation (Tokyo, Japan) as a donation. Test was performed according to the protocol of each manufacturer. The result was confirmed by at least two inspectors and adopted only in case with unanimous decision.

\section{Results}

The summary of patient's profile is presented in Table 1. All patients had pneumonia at admission, which was confirmed by chest CT examination. Among 26 patients with COVID19, 7 patients required mechanical ventilator support, which were designated as severe patients and one patient among them died because of multiple organ failure. The other 19 patients were named as moderate cases. As shown in Table 2 and Fig. 1, anti-SARS-CoV-2 IgG and IgM antibodies turned to be partially positive between the 6th and 10th days, more than $84 \%$ positive between the 11 th and 15 th days, and $100 \%$ after the 16 th day. When comparing three ELISA test kits, sensitivity of the anti-SARS-CoV-2 IgM of 2019-nCoV IgG/IgM Rapid Test Cassette was poorer than the other two kits. On the other hand, sensitivity of anti-SARS-CoV-2 IgG was comparable in three test kits. Data not shown, all of three test kits showed no false positive reaction in 12 negative control samples.

Table 3 presents the summary of moderate and severe patients. The severe patients were significantly older and had more elevated C-reactive protein at admission than those with moderate cases. On the contrary, qualitative trends of antiSARS-CoV-2 IgG and IgM antibodies were not different between both groups (Fig. 2; Tables 4 and 5).

Table 2 The positive ratio of anti-SARS-CoV-2 IgG and IgM antibodies detected by three different ELISA kits

\begin{tabular}{|c|c|c|c|c|c|}
\hline & $1 \sim 5$ days & $6 \sim 10$ days & 11 15 days & 16 20 days & 21 days $\sim$ \\
\hline \multicolumn{6}{|l|}{$\operatorname{IgM}$} \\
\hline AllTest & $0 / 1(0.00)$ & $0 / 10(0.00)$ & $5 / 17(0.25)$ & $2 / 9(0.22)$ & $2 / 6(0.33)$ \\
\hline Orient Gene & & $3 / 10(0.30)$ & $18 / 19(0.95)$ & 9/9 (1.00) & $6 / 6(1.00)$ \\
\hline INNOVITA & $0 / 1(0.00)$ & $2 / 10(0.20)$ & $16 / 19(0.84)$ & 9/9 (1.00) & $6 / 6(1.00)$ \\
\hline \multicolumn{6}{|l|}{$\operatorname{IgG}$} \\
\hline AllTest & $0 / 1(0.00)$ & $2 / 10(0.20)$ & $15 / 17(0.88)$ & 9/9 (1.00) & $6 / 6(1.00)$ \\
\hline Orient Gene & & $5 / 10(0.50)$ & $17 / 18(0.94)$ & $9 / 9(1.00)$ & $6 / 6(1.00)$ \\
\hline INNOVITA & $0 / 1(0.00)$ & $3 / 10(0.30)$ & $17 / 19(0.89)$ & $9 / 9(1.00)$ & $6 / 6(1.00)$ \\
\hline
\end{tabular}


Fig. 1 The trends of anti-SARSCoV-2 IgG and IgM antibodies detected by three different ELISA kits

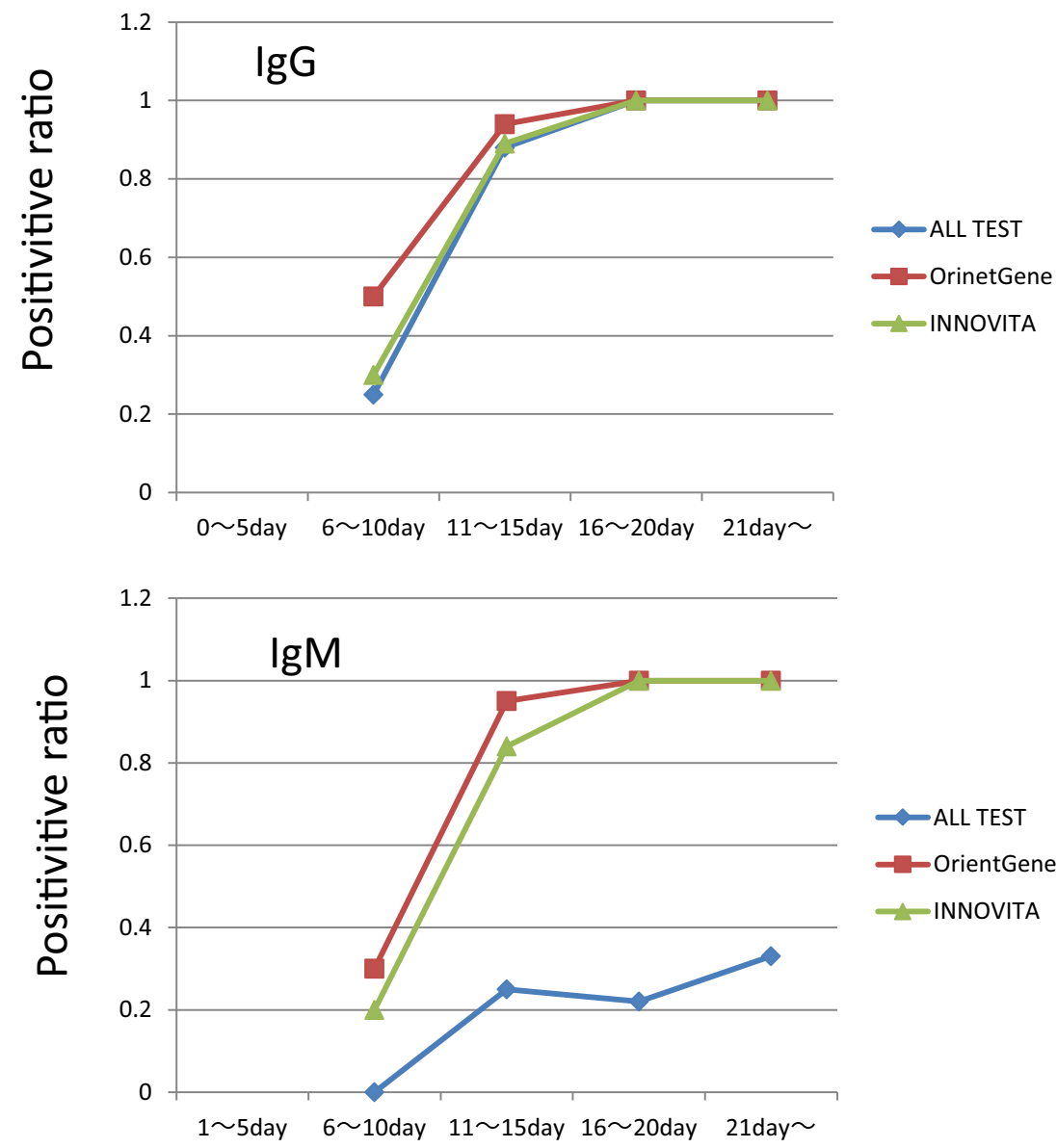

\section{Discussion}

The definitive diagnosis of COVID-19 is based on the detection of SARS-CoV-2 virus from nasopharynx, saliva, or stool by RT-PCR method. However, the diagnostic sensitivity of RT-PCR has been reported to be 70 to $80 \%$, which seems not sufficient enough to conduct a strict strategy for control of the infection prevalence, whereas the sensitivity of antibody test is reported to be approximately $100 \%$, although it requires 1 to 2 weeks after the initiation of illness that IgM and/or IgG antibody turns

Table 3 The profiles of severe and moderate patients

\begin{tabular}{llll}
\hline & Moderate & Severe & $P$ value \\
\hline Patient number & 19 & 7 & \\
Age (years) & $46.8 \pm 14.5$ & $68.4 \pm 9.1$ & $p=0.002^{*}$ \\
WBC $(/ \mu \mathrm{L})$ & 5416 & 7386 & $P=0.19$ \\
& \pm 1707 & \pm 3233 & \\
CRP $(\mathrm{mg} / \mathrm{dL})$ & $3.00 \pm 3.8$ & $16.5 \pm 7.2$ & $P=0.0004^{*}$ \\
Death & 0 & 1 & \\
\hline
\end{tabular}

Mann-Whitney $U$ test

*Statistically significant positive. In this sense, antibody test is not realistic for the diagnostic tool for the acute phase of infection. However, we have experienced a middle-aged man with characteristic pneumonia for COVID-19, who presented negative RT-PCR test for three times in a row. He showed a positive test for anti-SARS-CoV-2 IgG and IgM antibodies later and diagnosed as having COVID-19. In this way, antibody test might be sometimes useful as an indirect diagnostic tool for the sub-acute infection phase, clinically.

Most powerful utility of antibody test is an epidemiological analysis to conduct a proper and efficient strategy for controlling the pandemic of COVID-19. After the 1st wave of pandemic storm of COVID-19 in Japan, broad and precise epidemiological study by using the antibody test is expected to be done as early as possible.

Although our cohort is relatively small, this study presents valuable information for the COVID-19.

In our study, qualitative trends of anti-SARS-CoV-2 IgG and IgM antibodies were almost as same as previous reports from China and Singapore [3-6]. Interestingly, sensitivity of anti-SARS-CoV-2 IgG and IgM antibodies was $100 \%$ after 2 weeks in our cohort. 
INNOVITA
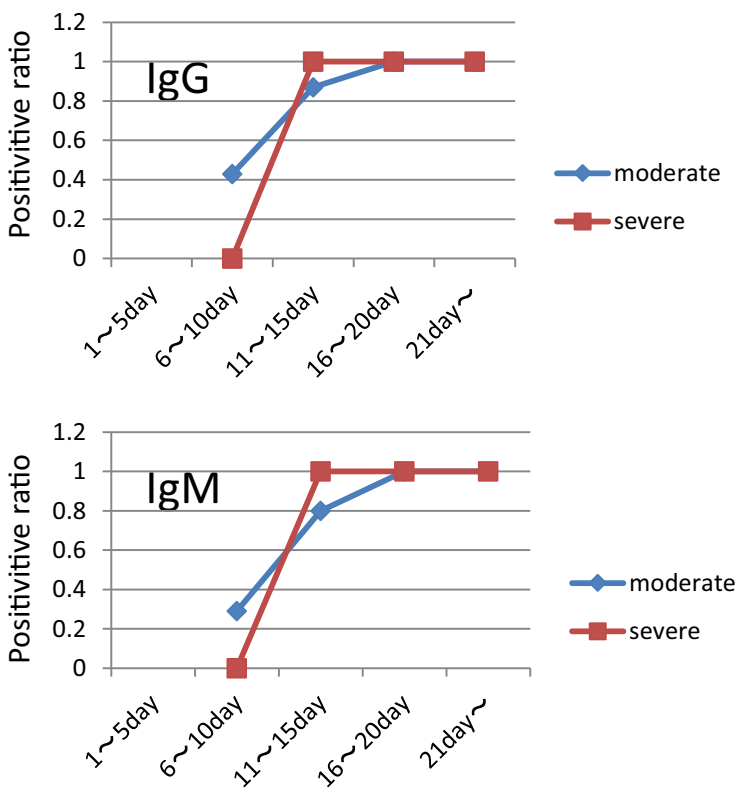

OrientGene
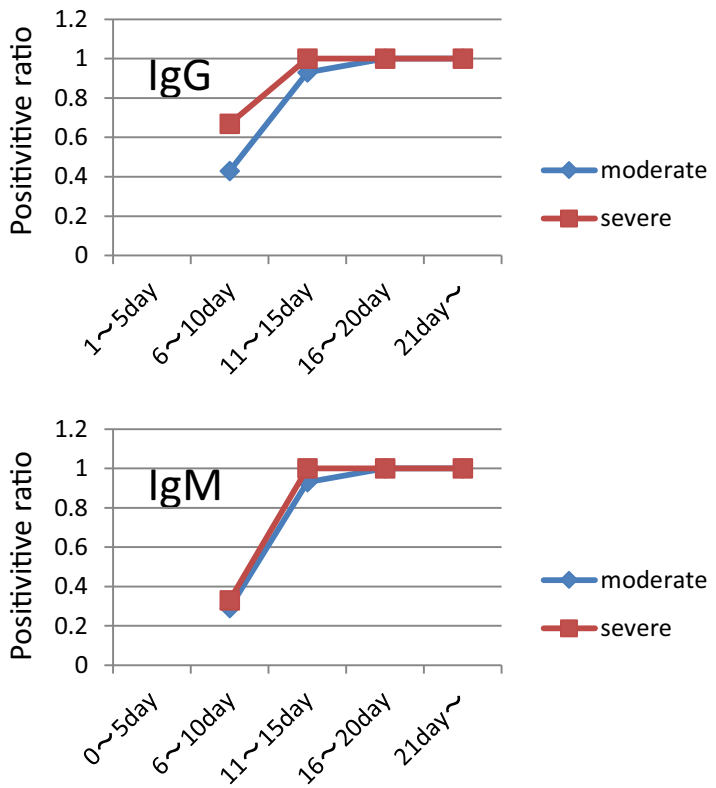

Fig. 2 Comparison of the trends of anti-SARS-CoV-2 IgG and IgM antibodies between severe and moderate cases

On the other hand, we have to be careful about the sensitivity of different ELISA kits, because some test kits may have poorer sensitivity like anti-SARS-CoV-2 IgM antibody of 2019-nCoV IgG/IgM Rapid Test Cassette (Hangzhou AllTest Biotech Co., China) in our study.

We could not observe the negative conversion of antiSARS-CoV-2 IgM antibody. It is considered that it requires at least 30 days for the negative conversion of anti-SARSCoV-2 IgM antibody.
Finally, we compared the trends of anti-SARS-CoV-2 $\operatorname{IgG}$ and IgM antibodies between severe and moderate cases and found no significant difference. However, we have to be careful about the interpretation, because antibody examined in this study does not necessarily reflect neutralizing activity, which is essential for the prevention of infection. It is required to investigate the neutralizing activity of antibody as well as the quantitative evaluation of antibody level to demonstrate the

Table 4 Comparison of positive ratio between severe and moderate cases detected by 2019-nCoV Ab Test (INNOVITA)

\begin{tabular}{|c|c|c|c|c|c|}
\hline & $1 \sim 5$ days & $6 \sim 10$ days & $11 \sim 15$ days & 16 20 days & 21 days $\sim$ \\
\hline \multicolumn{6}{|l|}{$\operatorname{IgM}$} \\
\hline Moderate & & $2 / 7(0.29)$ & $12 / 15(0.80)$ & 7/7 (1.00) & $2 / 2(1.00)$ \\
\hline Severe & $0 / 1(0.00)$ & $0 / 3(0.00)$ & $4 / 4(1.00)$ & $2 / 2(1.00)$ & $4 / 4(1.00)$ \\
\hline \multicolumn{6}{|l|}{$\mathrm{IgG}$} \\
\hline Moderate & & $3 / 7(0.43)$ & $13 / 15(0.87)$ & $7 / 7(1.00)$ & $2 / 2(1.00)$ \\
\hline Severe & $0 / 1(0.00)$ & $0 / 3(0.00)$ & $4 / 4(1.00)$ & $2 / 2(1.00)$ & $4 / 4(1.00)$ \\
\hline
\end{tabular}

Table 5 Comparison of positive ratio between severe and moderate cases detected by COVID-19 IgG/IgM Rapid Test Cassette (Orient Gene)

\begin{tabular}{|c|c|c|c|c|c|}
\hline & $1 \sim 5$ days & $6 \sim 10$ days & 11 15 days & 16 20 days & 21 days $\sim$ \\
\hline \multicolumn{6}{|l|}{$\operatorname{IgM}$} \\
\hline Moderate & & $2 / 7(0.29)$ & $14 / 15(0.93)$ & $7 / 7(1.00)$ & $2 / 2(1.00)$ \\
\hline Severe & & $1 / 3(0.33)$ & $4 / 4(1.00)$ & $2 / 2(1.00)$ & $4 / 4(1.00)$ \\
\hline \multicolumn{6}{|l|}{$\mathrm{IgG}$} \\
\hline Moderate & & $3 / 7(0.43)$ & $13 / 14(0.93)$ & $7 / 7(1.00)$ & $2 / 2(1.00)$ \\
\hline Severe & & $2 / 3(0.67)$ & $4 / 4(1.00)$ & $2 / 2(1.00)$ & $4 / 4(1.00)$ \\
\hline
\end{tabular}


precise difference of the immunological responses to SARS-CoV-2 infection between severe and moderate cases.

Acknowledgments The authors would like to express their deep gratitude to all of the medical staff of Musashino Red Cross Hospital, who dedicated themselves to take care of COVID-19 patients.

\section{Compliance with Ethical Standards}

Conflict of Interest The authors declare that they have no conflict of interest.

Ethical Approval This study was approved by the institutional review board as No2010.

Informed Consent Informed consent was secured by opt-out method.

\section{References}

1. Zhu N, Zhang D, Wang W, Li X, Yang B, Song J, et al. A novel coronavirus from patients with pneumonia in China, 2019. N Engl J
Med. 2020;382(8):727-33 Pubmed Central PMCID: PMC7092803. Epub 2020/01/25. eng.

2. World Health Organization Coronavirus disease (COVID-19). Situation report-140. https://wwwwhoint/docs/default-source/ coronaviruse/situation-reports/20200608-covid-19-sitrep-140pdf/ sfvrsn=2f310900_2 (accessed, June 9, 2020).

3. Zhang L, Pang R, Xue X, Bao J, Ye S, Dai Y, et al. Anti-SARS$\mathrm{CoV}-2$ virus antibody levels in convalescent plasma of six donors who have recovered from COVID-19. Aging (Albany NY). 2020;12 Epub 2020/04/23. eng.

4. Xiang F, Wang X, He X, Peng Z, Yang B, Zhang J, et al. Antibody detection and dynamic characteristics in patients with COVID-19. Clin Infect Dis. 2020;19 Epub 2020/04/20. eng.

5. Liu W, Liu L, Kou G, Zheng Y, Ding Y, Ni W, et al. Evaluation of nucleocapsid and spike protein-based ELISAs for detecting antibodies against SARS-CoV-2. J Clin Microbiol. 2020;30 Epub 2020/04/ 02. eng.

6. Yong SEF, Anderson DE, Wei WE, Pang J, Chia WN, Tan CW, et al. Connecting clusters of COVID-19: an epidemiological and serological investigation. Lancet Infect Dis. 2020;21 Pubmed Central PMCID: PMC7173813. Epub 2020/04/25. eng.

Publisher's Note Springer Nature remains neutral with regard to jurisdictional claims in published maps and institutional affiliations. 